\title{
Le titre de
}

\section{de l'équipage du souverain \\ Dr. Nevine Hussein Tolba}

Maître de conférence à la facultée de Lettres, l'Université d'Ain Chams nevinetolba@yahoo.com

Si le titre en question a tellement interessé les chercheurs cela est dû à ses nombreux translittérations et traductions.

Ce titre
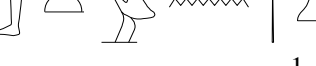

$\rightleftharpoons$ Atw

$\mathrm{n}$ Tt HqA selon Ward signifie, le serviteur de la table du roi. ${ }^{1}$ On a deux lectures de ce titre. La première est Wart signifiant <<partie >> ou <<région >> dérive du verbe war <<courir >>. D'après Faulkner, wartw n Tt HqA signifie contrôleur de la table du roi. C'est un officier en relation avec la table du souverain tandis que le récipiendaire est anx $n$ Tt HkA . ${ }^{2}$ En plus de la lecture war le signe se prononce AT ou At date du Moyen Empire dont le plus ancien exemple date

de Sesostris Ier. On trouve aussi un autre terme ce sont

les ATw qui remonte à l'Ancien Empire. Les anciens ATw étaient des tuteurs qui enseignaient les garçons tandis qu'au Moyen Empire, ATw ce titre était porté par les militaires. Ce mot ATw dérive du verbe AT qui signifie soigner ou protéger un enfant. On peut citer quatre exemples du mot ATw au Moyen Empire. ATyt la variante feminine de AT signifie nurse, ce titre est souvent mentionné près du titre des nourrices. ${ }^{3}$

Le dessin AT, et ATyt

Chevereau suggère que

signifie nurse. ${ }^{4}$ Tandis que matelots militaires. Ces personnes font parti de la flotte de guerre. Ce titre caractérise les membres de la marine au Moyen Empire. ${ }^{5}$ Ce n'est pas un titre

\footnotetext{
${ }^{1}$ Ward, W.(1982). Index of Egyptian Administrtive and Religious Titles of the Middle Kingdom, Beirut, p.7-8.

${ }^{2}$ Faulkner,R.O. (1988). A Concise Dictionary of Middle Egyptian, Oxford, p.58.

${ }^{3}$ Berlev,O.D. (1971).<<Les prétendus "citadins" du Moyen Empire>>, RdE 23,p.32.

${ }^{4}$ Faulkner, R.O. A Concise Dictionary of Middle Egyptian, p.6; Erman,A. \&Grapow,H.(1992).Wörterbuch der Ägyptischen Sprache I,23,9.

${ }^{5}$ Quirke, S.(2004). Titles and bureaux of Egypt,1850-1700 BC, GHP, p.99; Darnell, J.C. (2013). A Bureaucratic Challenge Archeology and Administration in Desert environement,
} 
spécifique d'un maître d'équipe mais tout officier appartenant à la flotte militaire. ${ }^{6}$ Le porteur du titre appartient à un groupe régulier du Moyen Empire et de la seconde période intermédiaire. Dans son article sur la marine durant le Moyen Empire, Berlev conclue que ce titre signifie les commandants de navires royaux sur les autres navires, et ne signifie pas le contrôleur ou le récipiendaire de la table du roi. ${ }^{7}$

\section{Les termes composés avec ATw:}

ATw $n$ ist commandant de la flotte, ATw aA $n$ niwt commandant en chef du régiment de la ville, ATw $n$ aAmw commandant des asiatiques, ATw $n$ wbAw commandant des porteurs d'aliments, ATw $n$ wrS commandant des gardiens, ATw n mniw Tsmw commandant des dresseurs des chiens, ATw $n$ niwt commandant du régiment de la ville, ATw $n$ kbnty commandant de la barque de Byblos, ATw n Tt nt pr-HD commandant des barques de la flotte du trésor ATw n Tt HqA commandant de la flotte royale. ${ }^{8}$

ATw $n$ nfww chef des matelots ${ }^{9}$, ATw $n$ ist chef d'équipage ${ }^{10}$, ATw $n$ aHaw commandant de vaisseau ${ }^{11}$.

Les termes composés avec wart:

wartw aA $n$ niwt chef administrateur de la ville ${ }^{12}$, wartw aA niwty chef administrateur des habitants de la ville ${ }^{13}$, wartw wHAt administrateur de l'oasis ${ }^{14}$.wartw $\mathrm{n}$ smyt administrateur du désert ${ }^{15}$, wartw $\mathrm{n}$ styw administrateur des nubiens. ${ }^{16}$

La participation à la marine royale était résensée aux hauts fonctionnaires et aux membres de la famille royale. La plupart d'entre eux portait ce titre. De

\footnotetext{
Handbook of Oriental Studies 104, Ancient Egyptian Administration in desert environment, p.812.

${ }^{6}$ Gardiner, A. (1938) <<The Mansion of Life and Master of King 's Largess >>, JEA 24,p.88 note 5 .

${ }^{7}$ Stefanovic, D.( 2009).The Non Royal Regular Feminine Titles of the Middle Kingdom and the Second Intermediate Period: Dossiers, GHP, p.90.

${ }^{8}$ Quirke,S. Titles and Bureaux,p.143.

${ }^{9}$ Chevereau,P.M.(1971). Prosopographie des cadres militaires, RdE 23, p.31.

${ }^{10}$ Ibid.,p.33.

${ }^{11}$ Ibid.,p.21.

${ }^{12}$ Ward,W. Index, p.84 n 695.

${ }^{13}$ Ibid.,p.84 n 696.

${ }^{14}$ Ibid.,p.84 n 697.

${ }^{15}$ Ibid.,p.84 n 700.

${ }^{16}$ Ibid.,p.84 n 701
} 
même, Quirke, traduit ce titre

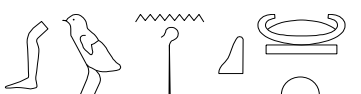

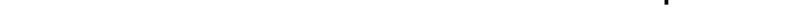
commandant de la flotte du roi" ce qui montre la haute position des ces titulaires dans la hiérarchie des commandants militaires de la fin du Moyen Empire et de la seconde période intérmédiaire comme c'est mentionné dans le papyrus Boulaq 18. ${ }^{17}$ Quirke, le met dans une position intermédiaire entre le commandant de l'armée imy-r mSawr et le commandant du régiment de la ville ATw aA $n$ Niwt $^{18}$. Même les femmes portaient le titre ATw $n \mathrm{Tt}$ HqA. ${ }^{19}$

\section{Xkrt nswt et sa relation avec ATw $n$ Tt HqA}

Ce terme trés répandu parmis les titres feminins du Moyen Empire, signifie la femme qui attend. ${ }^{20}$ Ce titre est porté par les femmes qui sont attachées à la cour royale à cause de leur relation familiale, Après le règne de Sobekhotep II , le titre est devenu plus populaire et il a remplacé nbt pr. On trouve 97 temoignages de ce titre durant le Moyen Empire.

On trouve une relation entre les Xkrt nswt les femmes qui attendent et les ATw n Tt HqA. Elles étaient les mères sur CGC 20322, CGC 20530, les femmes sur CGC 20668, et sur CGC 28030. ${ }^{22}$

\section{La raison du choix de translittération $A T w$}

Jj-m-jatw-jb travaillant durant le règne de Sobekhotep II. Le titre de cette personne s'écrit de deux manières. Dans ce cas, le $i$ est équivalant à $A, I T w$ est égale à ATw. Ce qui prouve que le titre doit être transcrit ATw n Tt HkA et non pas wart. ATw n'est connu qu'au Moyen Empire. ${ }^{23}$ La lecture de wartw est dérivé aussi du mot feminin wart qui signifie nurse tandis que le mot ATw masculin veut dire chef. ${ }^{24}$ On l'a vu une seconde fois dans le mot normal dans ATw $\mathrm{HqA} .^{25}$

ii-m-iai-ib est le propriétaire de la table d'offrandes d'El Amrah au Musée d'arts de Boston, le titre ATw n Tt HqA est écrit d'une façon

\footnotetext{
${ }^{17}$ Stefanovic, D. Non Royal Feminine Titles,p.90.

${ }^{18}$ Quirke,S. Titles and Bureaux, p.97.

${ }^{19}$ Stefanovic, D. Non Royal Feminine Titles, p.91.

${ }^{20}$ Stefanovic, D. (2009)<<Stela Bolton 1920.10.12〉>,SAK 38, p.299.

${ }^{21}$ Ibid.,p.300.

${ }^{22}$ Stefanovic, D.( 2006).Holders of Regular Military Titles in the Period of the Middle

Kingdom, GHP, Egyptology 4, London, p.90 n 406, 480, 462, 452, 417, 447, 428, 408, 449, 423, 460,436, 454,464,465; Franke,Personendaten, doss.718,312 a, 576,223,224,578

${ }^{23}$ Berlev, O. (1971).<<Les prétendus citadins du Moyen Empire〉>, RdE 23,p.32;

Chevereau, P.M. (1992). <<Contribution à la prosopographie des cadres militaires du Moyen Empire>>, RdE 43, p.23 n 413.

${ }^{24}$ Berlev,op.cit.,p.32.

${ }^{25}$ Ibid.,p.33.
} 
normale $^{26}$. Ainsi que le sceau de la collection Michaeldis et une stèle d' El Mahasna. Ses autres titres sont xtmw bity, $\mathrm{mr}$ gs $\mathrm{pr}^{27} \mathrm{Bbj}$ sur la stèle CGC 20524 datant de la XVII dynastie. ${ }^{28}$ Nfr Htp datant de la XIII dynastie. ${ }^{29}$

Weill mentionne dans son article de Recueil des Travaux qu'il accepte l'opinion de Spiegelberg que la translittération de ce titre demande un examen détaillé. Il s'est contenté de citer des exemples d'inscriptions de titres. Il a conclu que ce titre appartient à la XII dynastie ou la XIII dynastie et mentionne les différentes formes d'écriture. ${ }^{30}$ Les deux signes sont toujours au début mot tandis que la table d'offrandes suit le signe $\mathrm{HqA}$, quelquefois elle le prècéde. Selon Weill, cette table d'offrandes est une table royale. Les wartw Hqt sont des fonctionnaires importants. ${ }^{31}$

On trouve aussi les anxw HqA qui étaient "préposés aux barques des hiqitou du trèsor" qui figuraient sur les monuments. ${ }^{32} \mathrm{Il}$ a aussi mentionné l'existence d'un autre titre qui est wart aA n niwt. traduit Grand wartw de la ville. Ce titre ne peut pas éclairer la signification du titre précédent. Donc, pendant la XII dynastie, il y avait un service HqA "table royale", les anxw et les grands wartw de la ville. ${ }^{33}$

Sur une inscription du Ouadi Magharah mentionne le titre wartw n niwt et Brugsch l'a copié et le reproduit dans son thesaurus ${ }^{34}$ et Spiegelberg en 1899 le publia $^{35}$. Cette inscription se trouve dans le British Museum sur laquelle on trouve différentes formes d'écriture. Ce signe peut être remplacé par un autre signe qui n'a jamais existé dans aucune autre inscription de la XII dynastie. Il est possible que le scribe à Magharah ait confondu avec le mot de famille de wart qui n'a aucune relation avec notre titre. ${ }^{36}$

\footnotetext{
${ }^{26}$ Berlev,O. Les prétendus citadins, p.33.

${ }^{27}$ Chevereau, P.M. <<Contribution à la prosopographie des cadres militaires du Moyen Empire >, RdE 43(1992),p.23 n 413(PN I ,8,25).

${ }^{28}$ Franke,D.(1983). Personendaten aus dem Mittleren Reich,Wiesbaden, p162 dossier 223.

${ }^{29}$ Ibid.,p.210 dossier 312.

${ }^{30}$ Weill, R. (1905). <<Sur At.w-n-T.t-HqA et quelques titres analogues du Moyen Empire $>, R T 27$, p.41.

${ }^{31}$ Ibid.,p.42.

${ }^{32}$ Ibid.,p.43.

${ }^{33}$ Weill, R. (1905). $<<$ Sur At. $w-n-T \cdot t-H q A$ et quelques titres analogues du Moyen Empire >, RT 27,p.43.

${ }^{34}$ Brugsch,H.K. (1891)Thesaurus inscriptionum Aegyptiaerum, altaegyptische Inschriften (6), Bautexte und Inschriften verschiedenen Inhaltes altaegyptischer Denkmaeler , Leipzig, p. 1487.

${ }^{35}$ Spiegelberg,W. (1899). $<<$ Varia XLII $>>, R d T ~ 21$, p.51.

${ }^{36}$ Ibid.,p.44.
} 
Sur une table d'offrandes au nom d'anx-ni-f à Edfou datant de la XIII dynastie. On trouve ce titre traduit maître de l'autel". Sobekhotep était l'oncle d' $^{\prime}$ anx-ni-f. ${ }^{37}$

La traduction de titre par serviteur ou intendant de la table royale était suggèré par Ward, ATw n Tt HqA. ${ }^{38}$ De même que Wild ${ }^{39}$, Engelbach ${ }^{40}$ et Gardiner $^{41}$. Il apparait 50 fois dans les textes du Moyen Empire. Le gouverneur peut être le roi dans certains cas et peut être un officier ou nomarque dans d'autres cas. Les pères et les fils des titulaires étaient des officiers de classe inférieure intitulés "prêtre","serviteur de dieu", "prêtre lecteur". Donc l'intendant de la table royale était de niveau inférieur parmi les officiers administratifs. ${ }^{42}$ Leurs femmes portaient le titre de dame de maison ou femme qui attend. Ils servaient les gouverneurs locaux plus que les rois. ${ }^{43}$

La différence entre $\mathrm{Tt} \mathrm{HqA}$ et Tt niwt

Berlev a montré la différence entre Tt $\mathrm{HqA}$ et Tt niwt. Tt représente la flotte tandis que niwt représente le régiment terrestre de la ville mais Quirke trouve que le régiment de la ville dans la situation de la vallée du Nil n'est pas terrestre à moins qu'il demeure dans la ville. Selon les inscriptions des expéditions, on peut savoir que les régiments de la ville n'étaient pas attachés à leurs places d'origine. ${ }^{44}$ Sur les inscriptions d'Ouadi el Houdi, ce sont des groupes des troupes envoyées des différentes villes à une place particulière. C'étaient des représentants différents envoyés d'Elephantine, Kom Ombo et Thèbes ainsi que l'expédition de Senousert I à Ouadi el Houdi.

C'est pour cela que Quirke préfére opposer niwt HqA et considère Tt HqA comme l'étquipe du gouverneur. ${ }^{45}$

Quirke traduit ce titre Tt HqA comme l'équipe du gouverneur, les forces nationales tandis que niwt c'est le régiment urbain ou les forces locales. $\mathrm{Ce}$ titre c'est ATw n Tt HqA. Toutes les titres attachés aux ATw font partie des autorités de maintien de sécurité. ${ }^{46}$

\footnotetext{
${ }^{37}$ Edwards,A.B. (1888), <<The Provincial and Private collections of Egyptian Antiquities in Great Britain > , RdT 10, p.132.

${ }^{38}$ Ward, W. 1986. Essays on Feminine Titles of The Middle Kingdom and related Subjects, Beirut, p.115; Ward, Index, p.7 n 13.

${ }^{39}$ Wild, H.( 1871). <<Quatre statuettes du Moyen Empire dans une collection privée de Suisse $>, B I F A O 69$, p. 117 .

40 Engelbach, R.(1922). $<<$ Steles and Tables of Offerings of the Late Middle Kingdom from Tell Edfu $>, A S A E$ 22, p.119.

41 Gardiner,A.H.( 1938). <<The Mansion of Life and the Master of the King's Largess >>, JEA 24, p.88 n 5.

${ }^{42}$ Ward,W. Essays, p.115.

${ }^{43}$ Ibid.,p.116.

${ }^{44}$ Quirke,S. (1990).The Administration of Egypt in the Late Middle Kingdom, The Hieratic Documents, England, p.86 note 8.

${ }^{45}$ Quirke,S,Op.cit, p.82.

${ }^{46}$ Ibid.,p.83.
} 
Berlev l'avait transcrit ATw $\mathrm{n}$ Tt HqA.${ }^{47}$ Dans une inscription découverte dans la tombe de $R n(. i)$ snb à El $\operatorname{Kab}(n 9)$ datant de la XVII dynastie. C' était un soldat et un ATw n Tt HqA. Il était directement associé à la cour. ${ }^{48}$ C'était écrit sur une stèle à Edfou stèle d' ir .n.f. ${ }^{49}$ Ward et Edwards ${ }^{50}$ l'avaient traduite $\triangle \triangle \mathrm{mm}_{\triangle} \mathrm{ATw} \mathrm{n}$ Tt HqA l'intendant de la table royale. ${ }^{51}$ Mais Gardiner mentionne que l'officier en relation avec

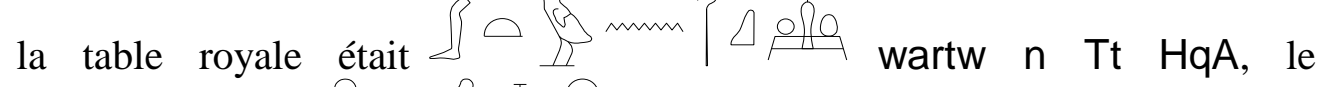
récipiendaire était anx $\mathrm{n}$ Tt HqA. ${ }^{52}$

Les officiers des navires

Ce sont des officiers des navires de guerre tandis que les anx n Tt HqA sont des matelots des navires de guerre comme le mentionnent les textes de Bouhen n 263, 881 et $1569 .^{53}$

Selon Faulkner, le vizir était aidé par le wartw du gouverneur et un conseiller d'armée qui lui donne des ordres. Dans l'expédition de Ramsès IV Ouadi Hammamat, une division compléte était employée l'officier qui commandait était le chef wartw de l'armée avec 20 wartw au dessous de lui, chacun commandant 250 hommes. ${ }^{54}$ Wartw $\mathrm{n}$ HqA peuvent être des officiers qui appliquent au vizir les charges militaires nécéssaires. ${ }^{55}$ Le grand nombre de wartw comparé aux récents enregistrements souligne une réorganisation de l'armée par Sethnakht ou Ramsès III après l'anarchie qui a terminé la XIX dynastie. wartw est un titre civil adapté pour des usages militaires. ${ }^{56}$

\footnotetext{
${ }^{47}$ Berlev, RdE 23,pp.23-38.

${ }^{48}$ Spalinger,A.J. (2002). A.Remarks on the family of queen Ha-s--nbw and the problem of kingship in dynasty ,JARCE 39.

${ }^{49}$ Alliot, M.( 1935). Rapport sur les fouilles de Tell Edfou(1933), FIFAO 10/2, p.34.

${ }^{50}$ Edwards, I. (1965).<<Lord Dufferin's Excavations at Deir el Bahari and the Clandeboye

Collection $>$, JEA 51, p.27.

${ }^{51}$ Ward, W.( 1982).Index of Administrative and Religious Titles of the Middle Kingdom,

Beirut,p.7 n 13.

${ }^{52}$ Gardiner, A.H.( 1938). <<The Mansion of Life and master of King's Largers >>, JEA 24, p.88-89 note5.

${ }^{53}$ Leprohon, R.J.( 1994). <<Maintien de l'ordre dans la Nubie au Moyen Empire〉>, Hommages à Jean Leclant vol 2 Nubie,Soudan,Ethiopie, BdE 106/2, p.288.

${ }^{54}$ Faulkner, R.O. (1953). <<Egyptian Military Organisation〉>, JEA 39, p.42.

${ }^{55}$ Ibid.,p.42 note 1.

${ }^{56}$ Ibid.,p.42 note7.
} 


\section{Les porteurs de ce titre durant le Moyen Empire}

Rn-snb datant dès la XIII-XVII dynastie. ${ }^{57} \mathrm{Hmn}-\mathrm{Htp}$ datant de la XIII dynastie, propriétaire de CCG $28126 .{ }^{58} \mathrm{Rn}$-snb datant de la XIII dynastie règne de Sobekhotep II. ${ }^{59}$ Hry datant de la XIII dynastie. ${ }^{60}$ sbk Htp datant de la XIII dynastie., propriétaire de CCG $20668^{61}$ Hwy-sbk était un compagnon d'armes de Sesostris III travaillait à l'époque du roi Ammenemes III. Il avait construit sa tombe à Abydos, et deux stèles à El Arabah au Musée de Manchester. Il a porté le titre ATw n Tt HqA la 9éme année d'Ammenemes III. Il est ainsi le maître de l'armée. La flotte fluviale avait des titres plus importants que l'armée terrestre. Il y avait des relations entre les ATw aA n niwt et les ATw n Tt HqA. ${ }^{62}$

sbk-Htp datant de la XIII dynastie, le propriétaire de la stèle JE $42201{ }^{63}$ snbw propriétaire d'une stèle à Edfou et de CGC 20294 datant de la XIII dynastie.. ${ }^{64} \mathrm{kms}$ datant de la XIII dynastie. ${ }^{65}$

AtHa selon une inscription dans une tombe à $1 \mathrm{El} \mathrm{Kab}$ tombe $\mathrm{n} 10$, datant du Moyen Empire et de la Seconde période intermédiaire. ${ }^{66}$ ly propriétaire de la stèle découverte à Bouhen et des inscriptions de la tombe d'El Kab n 10. ${ }^{67}$ II$\mathrm{m}$-iat-ib datant de la XIII dynastie au règne de Sobekhotep II sur une table d'offrandes découverte à Abydos, tombe n 62 à El Amrah ${ }^{68}$. ii-m-r possesseur d'une statue à Elephantine. ${ }^{69}$ ii.mrw datant de la XIII dynastie, fragment d'une stèle à Brussels E480 ${ }^{70}$,ii-mrt datant du Moyen Empire. laib,iatw, iw-snb, ${ }^{71} \mathrm{lpw}^{72}$,imny ${ }^{73}$,imny ${ }^{74}$, in-itf ${ }^{75}$,inpw ${ }^{76}$,iq-Htp ${ }^{77}$, iti

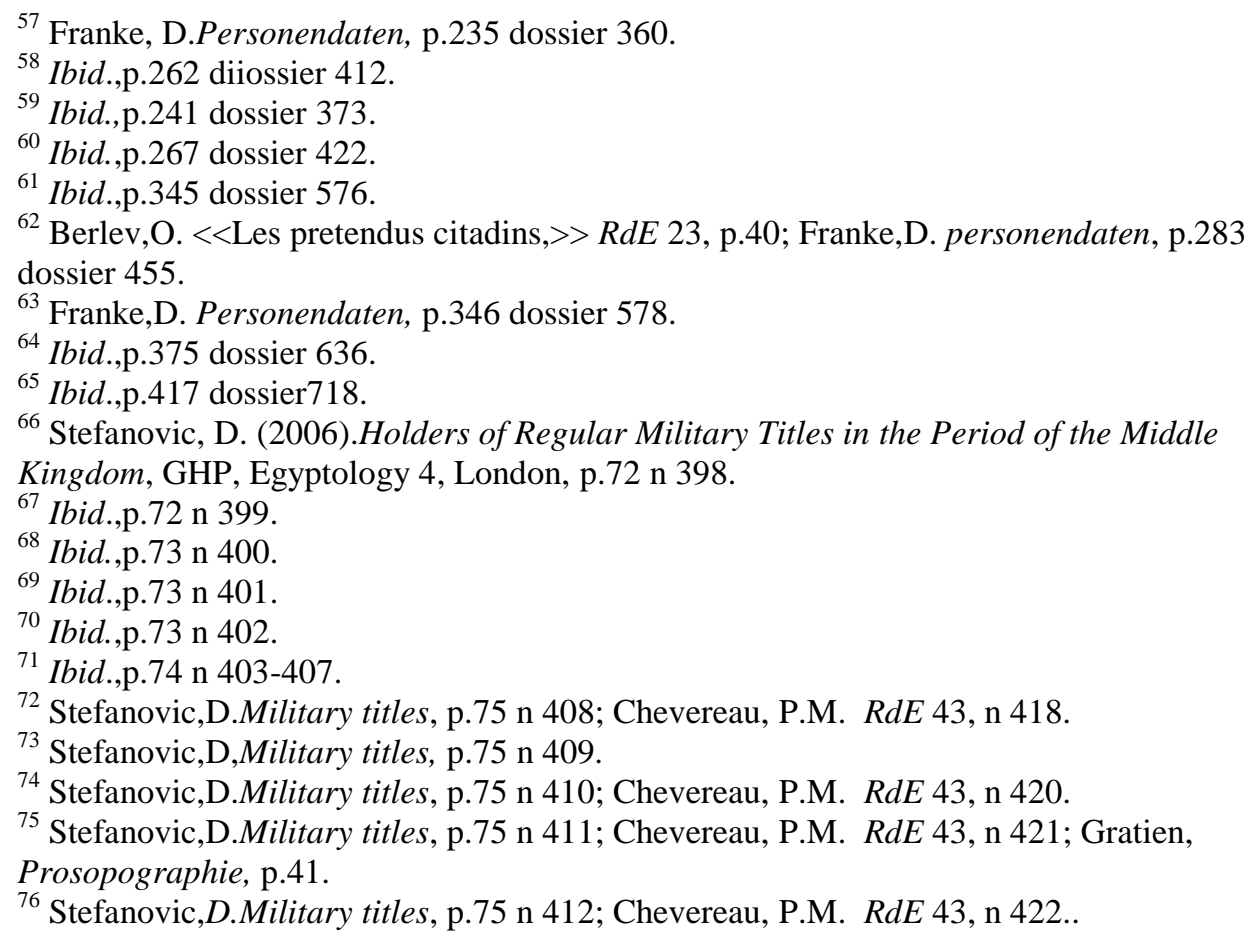


${ }^{78}$, it $^{79}$,anxw ${ }^{80}$, bbi,sanx ${ }^{81}$, ptH-Htp, ${ }^{82}$ mntw-m-HAt ${ }^{83}$, mnTw-Htp ${ }^{84}$, mry, ${ }^{85}$ ni-ib ${ }^{86}$, nb-iry-r-Aw ${ }^{87}$, nfr-Htp ${ }^{88}$.Nfrt-Htp.ty ${ }^{89}$, nsw. $^{90}$

ra-msw ${ }^{91}$, rn $^{92}$, rn-snb ${ }^{93}$.rn.f-rs ${ }^{94}$, rn.f-snb, ${ }^{95}$ rs $^{96}, \mathrm{Hmn}-\mathrm{Htp}{ }^{97}, \mathrm{Hr}-\mathrm{wn}-$

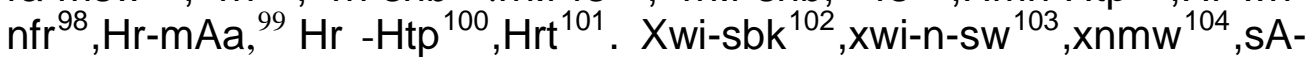
$\mathrm{iaH}^{105}$. sA-imn/rs ${ }^{106}, \mathrm{sA}-\mathrm{imn}^{107}, \mathrm{sA}-\mathrm{wnis}^{108}, \mathrm{sA}-\mathrm{HtHr}^{109}{ }^{10}$ sbk $^{110}$, sbk-mhAt ${ }^{111}$,sbk-m-Hb, sbk-m-sA.f. ${ }^{112}$ sbk-nxt ${ }^{113}$ sbk-Htp ${ }^{114}$, sn-rdiw ${ }^{115}$,

\footnotetext{
${ }^{77}$ Stefanovic,D.Military titles, p.75 n 413; Chevereau, P.M. RdE 43, n 423.

${ }^{78}$ Stefanovic,DMilitary titles, p.76 n 414.

${ }^{79}$ Stefanovic,D.Military titles, p.76 n 415; Chevereau, P.M. $R d E$ 43, n 423; Gratien, 58

${ }^{80}$ Stefanovic,D.Military titles, p.76 n 416; Chevereau, P.M. RdE 43, n 423; Gratien, 62.

${ }^{81}$ Stefanovic,D.Military titles, p.76 n 417; Chevereau, P.M. RdE 43, n 425.

${ }^{82}$ Stefanovic,D.Military titles, p.76 n 418; Chevereau, P.M. RdE 43, n 426.

${ }^{83}$ Stefanovic,D.Military titles, p.77 n 419; Chevereau, P.M. $R d E$ 43, n 427.

${ }^{84}$ Stefanovic,D.Military titles, p.77 n 420; Chevereau, P.M. $R d E$ 43, n 428.

${ }^{85}$ Stefanovic,D.Military titles, p.78 n 421; Chevereau, P.M. RdE 43, n 429.

${ }^{86}$ Stefanovic,D.Military titles, p.78 n 422; Chevereau, P.M. $R d E$ 43, n 430.

${ }^{87}$ Stefanovic,D.Military titles, p.78 n 423.

${ }^{88}$ Stefanovic,D.Military titles, p.78 n 424,425; Chevereau, P.M. RdE 43, n 431,432.

${ }^{89}$ Stefanovic,D.Military titles, p.79 n 426; Chevereau, P.M. $R d E 43, \mathrm{n} 433$.

${ }^{90}$ Stefanovic, D.Military titles, p.79 n 427; Chevereau, P.M. $R d E 43$, n 434.

${ }^{91}$ Stefanovic,D.Military titles, p.80 n 428; Chevereau, P.M. $R d E$ 43, n 435.

${ }^{92}$ Stefanovic,D.Military titles, p.80 n 429; Chevereau, P.M. $R d E 43$, n 438.

${ }^{93}$ Stefanovic,D.Military titles, p.80-81 n 430,431,432,433,434; Chevereau, P.M. RdE 43, n 439,440 .

${ }^{94}$ Stefanovic,D.Military titles, p.82 n 435.

${ }^{95}$ Stefanovic,D.Military titles, p.82 n 436; Chevereau,P.M. RdE 43, n 441.

${ }^{96}$ Stefanovic,D.Military titles, p.82 n 437; Chevereau, P.M. RdE 43, n 441.

${ }^{97}$ Stefanovic,D.Military titles, p.82 n 438; Chevereau, P.M. $R d E 43$, n 442.

${ }^{98}$ Stefanovic,D.Military titles, p.82 n 439; Chevereau, P.M. $R d E 43$, n 443.

${ }^{99}$ Stefanovic,D.Military titles, p.82 n 440; Chevereau, P.M. $R d E$ 43, n 444.

${ }^{100}$ Stefanovic,D.Military titles, p.83 n 441; Chevereau, P.M. RdE 43, n 445.

${ }^{101}$ Stefanovic,D.Military titles, p.83 n 442; Chevereau, P.M. RdE 43, n 446.

${ }^{102}$ Stefanovic,D.Military titles, p.84 n 443; Chevereau, P.M. RdE 43, n 447.

${ }^{103}$ Stefanovic,D.Military titles, p.84 n 444; Chevereau, P.M. RdE 43, n 448.

${ }^{104}$ Stefanovic,D.Military titles, p.84 n 445; Chevereau, P.M. RdE 43, n 449.

${ }^{105}$ Stefanovic,D.Military titles, p.84 n 446; Chevereau, P.M. RdE 43, n 450.

${ }^{106}$ Stefanovic,D.Military titles, p.85 n 448; Chevereau, P.M. RdE 43, n 451.

${ }^{107}$ Stefanovic,D.Military titles, p.85 n 449; Chevereau, P.M. RdE 43, n 452.

${ }^{108}$ Stefanovic,D.Military titles, p.85 n 450; Chevereau, P.M. RdE 43, n 453 ..

${ }^{109}$ Stefanovic,D.Military titles, p.86 n 452; Chevereau, P.M. RdE 43, n 454.

${ }^{110}$ Stefanovic,D.Military titles, p.86 n 453; Chevereau, P.M. RdE 43, n 455.

${ }^{111}$ Stefanovic,D.Military titles, p.86 n 454-455.

${ }^{112}$ Stefanovic,D.Military titles, p.86 n 457; Chevereau,P.M. RdE 43, n 456.

${ }^{113}$ Stefanovic, D.Military titles, p.87-88 n 458,459,461; Chevereau,P.M. RdE 43, n $457,458,459$.
} 
$\mathrm{snb}^{116}$,snbw ${ }^{117}$, snb. $^{118}, \mathrm{sbnw}^{119}, \mathrm{kAt}^{120}, \mathrm{kms}^{121}, \mathrm{kmsi}^{122}, \mathrm{t}-\mathrm{mr}^{123}$, $\mathrm{DwH}^{124}, \mathrm{Ddw}-\mathrm{Hr}$-pt-aty. ${ }^{125}$ Parmi les porteurs du titre on cite:AtH, iy, iyt, iim-iat-ib,ii-mr, ${ }^{126}$ ra-ib, iw-snb,ipw,imm.i,imnii,in(i) it-i-.f, inpw,iqHtp,it.. 127

idw-ami,anxw,bb-i,bb.i-sanx,ptH,mnTw-Htp,mrr.y,ni-ib,nfrHtp ${ }_{128}$,nsw,nDs-anx-iw,ra-ms(iw),rn.f-rs,rn-snb. ${ }^{129}$ rHw-rs, HmnHtp,Htr, $\quad$ xwi-wi-sbk,xnm.w. ${ }_{130}$ sA-iaH,sA-imn,sA-wnis,sA-xwt$\mathrm{Hr}$,sbk,sbk-m-HAt,sbk-m-sA.f,sbk-Htp, ${ }^{131}$ datant du Moyen Empire. sbkHtp,sn-wy,snb.w,snb.f,sn-rdi(w),Sbn.w,kbs..$^{132}$ datant de la XIII dynastie. kms,kmsi,kt,tA-mr,Tsw,ddw-Hr-pt-aty ${ }^{133}$ datant du Moyen Empire. ATw n ist chef d'équipage. ji-m-jatw-jb datant de Sobekhotep II. Bbj datant de la XVII dynastie ${ }^{134}$. Nfr-Htp datant de la XIII dynastie ${ }^{135}$. Rn-snb datant de la XVII dynastie ${ }^{136}$. Kms datant de la XIII dynastie. ${ }^{137}$

xwi-sbk datant d'Ammenemes III. ${ }^{138}$ snwsrt/wsi , aib-iaw. ${ }^{139}$ NDs-anx /lw datant de la XIII dynastie. CGC 20394. ${ }^{140}$ lyi fils de la maîtresse de

\footnotetext{
${ }^{114}$ Stefanovic,D. Military titles, p.88-89 n 462,463,464; Chevereau,P.M. RdE 43, n $460,461,462$.

${ }^{115}$ Stefanovic, D.Military titles, p.90 n 465; Chevereau, P.M. RdE 43, n 467.

${ }^{116}$ Stefanovic,D.Military titles, p.90 n 466.

${ }^{117}$ Stefanovic,D.Military titles, p.90 n 467; Chevereau, P.M. RdE 43, n 464,465.

${ }^{118}$ Stefanovic,D.Military titles, p.90 n 468; Chevereau, P.M. RdE 43, n 466.

${ }^{119}$ Stefanovic,D.Military titles, p.91 n 469; Chevereau, P.M. RdE 43, n 468.

${ }^{120}$ Stefanovic,D.Military titles, p.91 n 470; Chevereau,P.M. RdE 43, n 472.

${ }^{121}$ Stefanovic,D.Military titles, p.91 n 471; Chevereau,P.M. RdE 43, n 470.

${ }^{122}$ Stefanovic,D.Military titles, p.91 n 472; Chevereau,P.M. RdE 43, n 471.

${ }^{123}$ Stefanovic,D.Military titles, p.91 n 473; Chevereau,P.M. RdE 43, n 473.

${ }^{124}$ Stefanovic,D.Military titles, p.91 n 474.

${ }^{125}$ Stefanovic,D.Military titles, p.91 n 475; Chevereau,P.M. RdE 43, n 475.

${ }^{126}$ Chevereau, P.M.( 1992).<< Contribution à la prosopographie des cadres militaires du Moyen Empire>>, RdE 43, Paris,p.23 n 411-414.

${ }^{127}$ Ibid.,p.23 n 415-423.

${ }^{128}$ Chevereau, op.cit.,p. 25 n 423-432.

${ }^{129}$ Ibid.,p.26 n 433-440.

${ }^{130}$ Ibid.,p.27 n 441-449.

${ }^{131}$ Ibid.,p. 28 n 450-460.

${ }^{132}$ Ibid.,p.29 n 461-469.

${ }^{133}$ Chevereau, op.cit.,,p.30 n 470-480; Quirke,S. Titles and Bureaux, p.97.

${ }^{134}$ Franke,D. Personendaten, p.53 dossier 23.

${ }^{135}$ Franke,D. Personendaten,p.162 dossier 312.

${ }^{136}$ Ibid.,p. 235 dossier 360.

${ }^{137}$ Ibid.,p.417 dossier 718 .

${ }^{138}$ Vernus, P. (1986).Le Surnom au Moyen Empire, repertoire procédés d'Expression et Structures de la double Identité du début de la XIIe Dynastie à la Fin de La XVIIe

Dynastie,BIP, Rome,p.44 n 192.

${ }^{139}$ Ibid.,p. 48 n 211.
} 
maison didit fin de la XIII dynastie. ${ }^{141}$ In i-it,-. ${ }^{142}$ datant de la XII dynastie. ${ }^{143}$ anxw, ${ }^{144}$ It et aidw-ami. ${ }^{145}$ xwi-sbk, hw(i)-sbk ${ }^{146}$, datant de la XII dynastie. ${ }^{147} \mathrm{xn}, \mathrm{xnmw}$, datant du Moyen Empire. Durant l'Ancien Empire le commandant des équipages de recrues était appelé xrp aprw nfrw ${ }^{148}$ $\Longrightarrow$ Mr aprw chef d'équipage ${ }^{149}$. Selon Lesko, pendant le

Nouvel Empire, 2 AT signifie administrateur ou intendant. ${ }^{150}$ Wolterman l'avait transcrit ATw et donne exemple ATw $n$ imnt niwt qui avait plus de responsabilité que le maire de Thèbes Ouest. ${ }^{151}$

Parmi les termes qui se composent avec Tt HqA

bAqt nt Tt HqA, anxt nt Tt HqA. Franke l'a transcrit ATw n Tt HqA ${ }^{152}$ sur la stèle de Rnsnb ${ }^{153}$, sur la stèle d'Edfou de Senebou, il porte le même titre. ${ }^{154}$ Gardiner, trouve que ce signe doit être transcrit wart puisque la ville est appellée HAt wart imntt de l'Ouest, la nome libyenne à l'extrémité du Delta. La transcription du signe wart est un probléme, il est parfois transcrit wart parfois ATw. Il signifie une division administrative, quartier, district, département. Mais Posener, suggère une autre lecture des deux signes

en se basant sur la graphie

Litée dès la VI dynastie jusqu'à la XII dynastie. ${ }^{155}$ Les deux premiers signes ont le déterminitatif de la jambe peut signifier éducateur ou administrateur. La présence de ce

\footnotetext{
${ }^{140}$ Ibid.,p.75 n 346.

${ }^{141}$ Gratien,B.( 1951). Prosopographie des Nubiens et des Egyptiens en Nubie durant le Moyen Empire, CRIPE Lille,p.24.

${ }^{142}$ Ibid.,p.41.

${ }^{143}$ Ibid.,p.62.

${ }^{144}$ Ibid.,p.58.

${ }^{145}$ Ibid.,p.136.

${ }^{146}$ Ibid.,p.137.

${ }^{147}$ Ibid.,p.140.

${ }^{148}$ Baud, M.( 1999). <<Famille royale et pouvoir sous l'Ancien Empire >>, BdE126/1, p.278.

${ }^{149}$ Chevereau, P.M.( 1992). $<<$ Contribution à la prosopographie des cadres militaires du

Moyen Empire >, RdE 43, Paris,p.31.

${ }^{150}$ Lesko,L.H. (2002). Dictionary of Late Egyptian vol.I, p.10.

${ }^{151}$ Wolterman, C.( 1996). <<A vizir of Ramses III visits an oracle >>, RdE 47, Paris p.161.

${ }^{152}$ Franke, D.( 1990). <<Reviews of Ward Essays on Feminine Titles of the Middle

Kingdom and Related Subjects >, JEA 76, p.229.

${ }^{153}$ Ibid.,p. 230.

${ }^{154}$ Kuentz, M.C. (1923). <<Deux stèles d'Edfou〉>, BIFAO 21, p.108.

${ }^{155}$ Wild, H.( 1971). $<<$ Quatre statuettes du Moyen Empire dans une collection privée de Suisse $>, B I F A O$ 69, p.117.
} 
déterminatif dans le verbe mentionné deux fois dans le papyrus Westcar confirme cette opinion. ${ }^{156}$

Ce titre est traduit ATw ayant apparu dès la VI dynastie jusqu'

à la XII dynastie. Il est équivalant à $\triangle$ ATw et signifie contrôleur, surveillant, administrateur ainsi qu'intendant. Ces signes ont apparu sous la XII dynastie.Ces mots montrent l'organisation administrative pendant le Moyen Empire. Tandis qu'au Nouvel Empire c'était un titre militaire. ${ }^{157}$

D'après Wild, ATw $\mathrm{n}$ Tt HqA signifie intendant de la table du souverain, contrôleur des commensaux du souverain. ${ }^{158}$ Selon Gardiner, l'officier responsable des commensaux (gens de table) la table royale est un intendant responsable de la bouche du palais. ${ }^{159}$ Wild a classifié les officiers selon leurs rôles dans les services de la résidence.:

Les mots en relation avec ATw:

ATw n Tt HqA "intendant de la table royale", ATw wbAw" intendant des échansons" ,ATw n smsw"intendant des suivants" , ATw n niw Tsmw intendant des dresseurs des chiens ${ }^{160}$. ATw $n$ Tt nt pr-HD "intendant de la personne du trèsor". ATw $\mathrm{n}$ wrSw "intendant de la surveillance de la

journée", $\quad$ ATw aA n niwt "administrateur de la ville", ATw aA n niwtyw "grand administrateur des citoyens", ATw n niwt rsyt wart mHtt "administrateur du secteur nord de la ville sud". ${ }^{161}$

idnw ATw $n$ Mn-nfr substitut des administrations de Memphis. idnw $n$ ATw $n$ tA iwyt mHtt substitut de l'administrateur du quartier septentrional, ATw $n$ smyt surveillant de la nécropole, ATw $n$ wHAt surveillant ou administrateur de l'oasis ATw $n$ stywt surveillant des nubiens, ATw n pA mSa intendant de l'armée. ${ }^{162}$

Durant le Moyen Empire, $\int \subset \_$mm $\{\Delta$ wartw $\mathrm{n}$ Tt HqA était l'officier responsable de la table royale et le recipiendaire est appelle

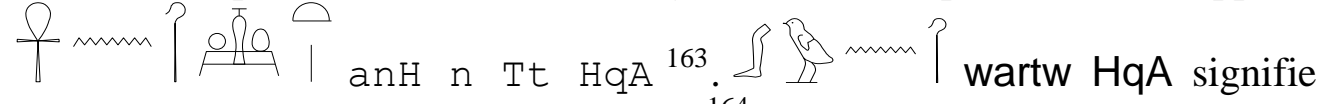
l'officier du roi. Il jouait un rôle militaire. ${ }^{164}$

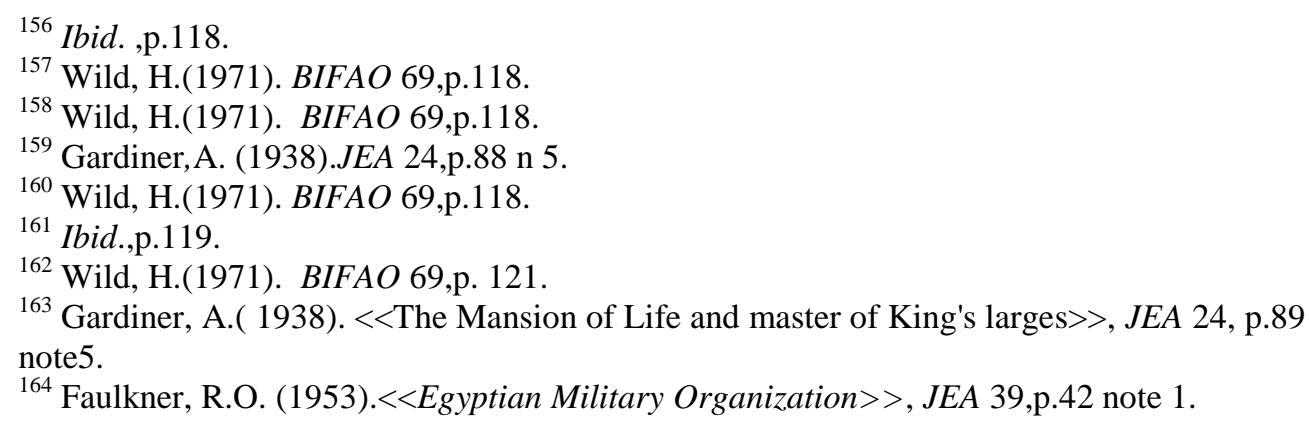


Les officiers étaient organisés dès la XII dynastie jusqu'à la fin de la XIII dynastie. réunis dans des secteurs ouvrièrs comme El Lahoun à Fayoum, et Thèbes. Ces secteurs étaient lu wart et les grandes divisions administratives du pays ainsi que les chefs de bijoutiers, des artisans du métal., des peintres, des sculpteurs, des tailleurs de pierre qui les construisaient. ${ }^{165}$ Les porteurs du titre

ATw surveillaient les rouages de l'administration qui était

auparavant l'occupation des anciens

ATw sous la XI dynastie. Ils étaient aussi les administrateurs de ville ce qui correspond avec le terme feminin Atyt qui est souvent associé avec mnat nourrice. Donc les $\left.\int\right]$ ATw peut aussi signifier maître de quartier. ${ }^{166}$

$\operatorname{Itw}(\mathrm{t})$ est une variante de Atwt $\langle<$ couche $\rangle\rangle,\langle\langle$ siège $\rangle\rangle$. Ce mot est similaire à $A T w t<<$ nurse $>>$ qui est le pendant feminin de ATw. On trouve une relation entre le lit ou le banc et la personne couchée dessus dont le lit en prend soin. Puisque les pieds du lit prenaient la forme de pattes de vache ou de lionne, les Egyptiens faisaient allusion aux déesses-éducatrices ou ils symbolisaient l'animal divinisé. ${ }^{167}$

Selon Fischer, ce signe $\perp$ est transcrit wart. Il le confirme parce que T.G.H.James transcrit la stèle de BM 573, il l'a même traduit le grand administrateur mais non pas dans la grande ville puisque le nom de la ville est écrite différemment. ${ }^{168}$ Fischer accepte l'opinion de Franke à propos de ce signe qui ne doit pas avoir deux lectures différentes. Il doit être traduit assistant. Pour le choix ATw et wart, il a choisi la lecture selon la stèle de BM 573 qui l'avait traduit wart qui l'assimile le signe signifiant grand administrateur. Selon Grapow, avec war Tt HqA chef de la table du souverain date du Moyen Empire. $\int \subset{ }_{\text {wartw }}$ signifie chef apparu dès le Moyen Empire ${ }^{169}$ et au Nouvel Empire. ${ }^{170}$ Selon Faulkner, wartw signifie administrateur civil wartw n Tt HqA contrôleur de

\footnotetext{
${ }^{165}$ Wild, op.cit.,p.119.

${ }^{166}$ Wild,op.cit.,p. 121.

${ }^{167}$ Berlev, O.<<les pretendus citadins >>, p.33 note 1.

${ }^{168}$ Fischer, H.G.(1985).Egyptian Titles of The Middle Kingdom, A supplement to W. Ward's Index, Metropolitain Museum of Art, p.99.

${ }^{169} \mathrm{~Wb} I, 288,13$.

${ }^{170} \mathrm{~Wb} I, 288,9$.
} 
la table du souverain. ${ }^{171}$ Franke donne un exemple où $\left.\int\right\} \subseteq$ est remplacé par ${ }^{\stackrel{\Omega}{\Omega}}$ comme écriture de Atw. ${ }^{172}$ Dans ce cas iTw est égale à ATw.

Le signe est transcrit wart par Gardiner. ${ }^{173}$ wartw est dérivé du mot wart

II signifiant division administrative comme <<quartier, département〉> ou grande division. ${ }^{174}$

Chevereau l'a transcrit ATw et

ATw n Tt HqA l'a traduit chef des matelots militaires tandis que Berlev l'a traduit chef de l'équipage du souverain. Ces personnels appartiennent à la flotte de guerre. ${ }^{175}$ Parmi les porteurs du titre on cite:AtH, iy, iyt, ii-m-iat-ib,ii-mr, ${ }^{176}$ ra-ib, iwsnb,ipw,imm.i,imnii,in(i) it-i-.f, inpw,iq-Htp,it..177

idw-ami,anxw,bb-i,bb.i-sanx,ptH,mnTw-Htp,mrr.y,ni-ib,nfrHtp 178 ,nsw,nDs-anx-iw,ra-ms(iw),rn.f-rs,rn-snb. ${ }_{180}^{179}$ rHw-rs, HmnHtp,Htr, $\quad$ xwi-wi-sbk,xnm.w. ${ }_{180}$ sA-iaH,sA-imn,sA-wnis,sA-xwtHr,sbk,sbk-m-HAt,sbk-m-sA.f,sbk-Htp,${ }^{181}$ datant du Moyen Empire. sbkHtp,sn-wy,snb.w,snb.f,sn-rdi(w),Sbn.w,kbs. i $^{182}$ datant de la XIII dynastie. kms,kmsi,kt,tA-mr,Tsw,ddw-Hr-pt-aty ${ }^{183}$ datant du Moyen Empire. ATw n ist chef d'équipage. ji-m-jatw-jb datant de Sobekhotep II. Bbj datant de la XVII dynastie ${ }^{184}$. Nfr-Htp datant de la XIII dynastie ${ }^{185}$. Rn-snb datant de la XVII dynastie ${ }^{186}$. Kms datant de la XIII dynastie. ${ }^{187}$

\footnotetext{
${ }^{171}$ Faulkner,R.O. A Concise Dictionary, p. 58.

${ }^{172}$ Fischer, H.G.(1985).Egyptian Titles of The Middle Kingdom,p.39 n 2.

${ }^{173}$ Gardiner, A.H.p. 100.

${ }^{174}$ Wild, op.cit.,p.117.

${ }^{175}$ Chevereau, P.M.( 1992). << Contribution à la prosopographie des cadres militaires du Moyen Empire>>, RdE 43, Paris,p.23 note 23.

${ }^{176}$ Chevereau, P.M.( 1992).<< Contribution à la prosopographie des cadres militaires du Moyen Empire>>, RdE 43, Paris,p.23 n 411-414.

${ }^{177}$ Ibid.,p.23 n 415-423.

${ }^{178}$ Chevereau, P.M. op.cit.,p. 25 n 423-432.

${ }^{179}$ Ibid.,p.26 n 433-440.

${ }^{180}$ Ibid.,p.27 n 441-449.

${ }^{181}$ Ibid.,p. 28 n 450-460.

${ }^{182}$ Ibid.,p.29 n 461-469.

${ }^{183}$ Chevereau,P.M. op.cit.,,p.30 n 470-480; Quirke, S.Titles and Bureaux, p.97.

${ }^{184}$ Franke,D. Personendaten, p.53 dossier 23.

${ }^{185}$ Franke,D. Personendaten,p. 162 dossier 312

${ }^{186}$ Ibid.,p. 235 dossier 360.

${ }^{187}$ Ibid.,p.417 dossier 718.
} 
xwi-sbk datant d'Ammenemes III. ${ }^{188}$ snwsrt/wsi, aib-iaw. ${ }^{189}$ NDs-anx /lw datant de la XIII dynastie. CGC 20394. ${ }^{190}$ lyi fils de la maîtresse de maison didit fin de la XIII dynastie. ${ }^{191}$ In i-it,-.f ${ }^{192}$ datant de la XII dynastie. ${ }^{193}$ anxw, ${ }^{194}$ It et aidw-ami. ${ }^{195}$ xwi-sbk, hw(i)-sbk ${ }^{196}$, datant de la XII dynastie. ${ }^{197} \mathrm{xn}$, xnmw, datant du Moyen Empire. Durant l'Ancien Empire le commandant des équipages des recrues était appelé xrp aprw nfrw. ${ }^{198}$ Selon Van den Boorn, ce titre doit être traduit le capitaine de l'équipage du souverain comme Berlev l'a prouvé. Parfois la table est pleine d'offrandes, d'autres fois non. ${ }^{199}$

Durant le Nouvel Empire, ce titre marin montre le statut spécial qu'il avait, il a joué un rôle important au début du Nouvel Empire dans la libération durant le règne de Thoutmosis et Ramsès II surtout en Syrie. Ainsi, au début de la XVIII dynastie dans la guerre et dans la préparation de l'armée. Il devient d'une grande importance ce que le montre le papyrus Anstasi où on trouve, sous Ramsès III, les troupes, les chariots et la flotte sur le lac. Durant le règne de Piankhi, des inscriptions décrivent le déroulement de la guerre.

Ce titre peut aussi signifier Les responsables de la table du souverain. Ils étaient nombreux durant le Moyen Empire et on ne spécifie pas leurs activités. wartw $n \mathrm{Tt} \mathrm{HqA}$. Le déterminatif qui l'accompagne est une table et offrandes ainsi le titre doit être lu comme serviteur de la table du souverain. Ce titre se lit ATw n Tt HqA selon Helck dans le papyrus Boulaq.

D'après le papyrus Boulaq, les ATw njw Tt HqA étaient les officiers de la flotte fluviale de la guerre. Ces officiers étaient supérieurs aux porteurs de titres de l'armée terrestre. ${ }^{200}$ Dans un texte datant

${ }^{188}$ Vernus,P.(1986).Le Surnom au Moyen Empire, procédés d'expression et structures de la double identité du début de la XIIe dynastie a la fin de la XVIIe dynastie,BIP, Rome. P.44 n 192.

${ }^{189}$ Ibid.,p.48 n 211.

${ }^{190}$ Ibid.,p.75 n 346.

${ }^{191}$ Gratien,B.( 1951). Prosopographie des Nubiens et des Egyptiens en Nubie durant le

Moyen Empire, CRIPE Lille,p.24.

192 Ibid.,p.41.

193 Ibid.,p.62.

${ }^{194}$ Ibid.,p.58.

195 Ibid.,p. 136.

${ }^{196}$ Ibid.,p. 137.

${ }^{197}$ Ibid.,p. 140.

${ }^{198}$ Baud, M.( 1999).<<Famille royale et pouvoir sous l'Ancien Empire 〉>, BdE126/1, p.278.

${ }^{199}$ Boorn, Van den. (1988).The Duties of the Vizir, New York,p.225.

200 Berlev,O. citadins,p.40 n 6. p.Boulaq XVIII, 26,1-6,27,2,12 
du Moyen Empire copié durant la XVIII dynastie, on connait "l'emploi du temps du vizir". Le vizir, chef de l'administration égyptienne, appelait les ATw w njw Tt HqA et la DADAt njt mSa pour leur donner les consignes concernant l'armée. $^{201}$ Ils étaient aussi nommés les wartw que le roi appelle pour leur donner ses ordres. ${ }^{202}$

Donc, ce titre a deux translittérations ATw ou wart ainsi que des traductions différentes comme le serviteur de la table du roi, l'intendant de la table royale, le commandant de navires royaux, le commandant de la flotte du roi, capitaine de l'équipage du souverain, chef des matelots militaires. La traduction du titre dépend du déterminatif qui l'accompagne. Lorsque le déterminatif est une table d'offrandes le titre est traduit le serviteur de la table du roi mais si le déterminatif est une navire le titre est traduit le commandant de la flotte du roi. C'est très difficile de préciser la traduction exacte de ce titre comme l'ont sugggéré les différents chercheurs.

\section{Liste des référemces et des périodiques:}

- Alliot, M.( 1935). Rapport sur les fouilles de Tell Edfou(1933), Fouilles de l' l'Institut Francais d'Archéologie Orientale 10/2, p.34.

Baud, M.( 1999). <<Famille royale et pouvoir sous l'Ancien Empire >>, BdE126/1, p.278.

Berlev,O.D. (1971).<<Les prétendus "citadins" du Moyen Empire〉>, Revue d'Egyptologie 23, pp.23-38,40-41.

Boorn,Van den. (1988).The Duties of the Vizir, New York,p.225.

-Brugsch,H.K.(1891).Thesaurus Inscriptionum Aegyptiaerum, Altaegyptische Inschriften (6), Bautexte und Inschriften verschiedenen Inhaltes altaegyptischer Denkmaeler, Leipzig, p.1487.

Chevereau, P.M.( 1992). << Contribution à la prosopographie des cadres militaires du Moyen Empire>>, Revue d'Egyptologie 43, Paris,p.23,24 n 411423, p.25 n 423-432, p.26 n 433-440, p.27 n 441-449, p.28 n 450-460, p.29 n 461-469, p.30 n 470-480,p.31.

- Darnell, J.C. (2013). A Bureaucratic Challenge Archeology and Administration in Desert environement, Handbook of Oriental Studies 104, Ancient Egyptian Administration in desert environment,p.812.

Edwards, I. (1965). $<<$ Lord Dufferin's Excavations at Deir el Bahari and the Clandeboye Collection>>, Journal of Egyptian Archeology 51, p.27.

Edwards,A.B. (1888), <<The Provincial and Private collections of Egyptian Antiquities in Great Britain〉>, Recueil des Travaux 10, p.132.

${ }^{201}$ Berlev,O. citadins,p.41 n 9.
${ }^{202}$ Faulkner,R,O.( 1953). Egyptian Military Organization, JEA 39, p.43. 
-Engelbach, R.(1922). $<<$ Steles and Tables of Offerings of the Late Middle Kingdom from Tell Edfu>>, Annales du Service des Antiquités Egyptiennes 22, p.119.

Erman,A. \& Grapow,H.(1992).Wörterbuch der Ägyptischen Sprache I,23,9, 288,9,288,13.

Faulkner,R.O. (1988). A Concise Dictionary of Middle Egyptian, Oxford, p.6 p.58.

-Faulkner, R.O. (1953). <<Egyptian Military Organization>>, Journal of Egyptian Archeology 39, p.42 note 1, note 7.

-Fischer, H.G.(1985).Egyptian Titles of The Middle Kingdom, A supplement to W. Ward's Index, Metropolitain Museum of Art, pp.39 n 2,99.

-Franke,D.(1983). Personendaten aus dem Mittleren Reich,Wiesbaden,, pp.53,162,210,235, 241, 262, 267, 283, 345,346,375, 417.

Franke, D.( 1990). <<Reviews of Ward Essays on Feminine Titles of the Middle Kingdom and Related Subjects >>, Journal of Egyptian Archeology 76, pp.229-230.

Gardiner, A. (1938) <<The Mansion of Life and Master of King 's Largess >>, Journal of Egyptian Archeology 24, pp.88-89 note 5, 100.

Gratien,B.( 1951). Prosopographie des Nubiens et des Egyptiens en Nubie durant le Moyen Empire, CRIPE Lille,pp.24, 41, 62, 58, 136-7, 140.

Kuentz, M.C. (1923). <<Deux stèles d'Edfou>>, Bulletin de l'Institut Francais d'Archéologie Orientale 21, p.108.

Leprohon, R.J.( 1994).<<Maintien de l'ordre dans la Nubie au Moyen Empire>>, Hommages à Jean Leclant vol 2 Nubie, Soudan, Ethiopie, Bibliothèque d'Etude 106/2, p.288.

Lesko, L.H. (2002). Dictionary of Late Egyptian vol.I, p.10.

Quirke,S. (1990).The Administration of Egypt in the Late Middle Kingdom, The Hieratic Documents, England, pp.82, 86 note 8.

Quirke, S.(2004). Titles and bureaux of Egypt, 1850-1700 BC, Golden House Publications, pp.97,99,143.

Spalinger,A.J. (2002). A.Remarks on the family of queen Ha-s--nbw and the problem of kingship in dynasty, Journal of American Research Center in Egypt 39.

- Spiegelberg,W. (1899).<<Varia XLII >>, Recueil des Travaux 21,pp.44, 51. Wild, H.( 1971). <<Quatre statuettes du Moyen Empire dans une collection privée de Suisse >, Bulletin de l'Institut Francais d'Archéologie Orientale 69, pp.117-121.

- Stefanovic, D.(2006).Holders of Regular Military Titles in the Period of the Middle Kingdom, Golden House Publications, Egyptology 4, London, pp.72 ,75-91, 90 n 406, 480, 462, 452, 417, 447, 428, 408, 449, 423, 460,436, $454,464,465$ 
- Stefanovic, D. ( 2009)<<Stela Bolton 1920.10.12〉>,SAK 38, pp.299,300. -Stefanovic, D.( 2009).The Non Royal Regular Feminine Titles of the Middle Kingdom and the Second Intermediate Period: Dossiers, Golden House Publications, pp.90,91.

- Vernus,P.(1986).Le Surnom au Moyen Empire, procédés d'expression et structures de la double identité du début de la XIIe dynastie à la fin de la XVIIe dynastie,BIP, Rome. pp.44 n 192, 48 n 211, 75 n 346.

Ward, W. (1986). Essays on Feminine Titles of The Middle Kingdom and related Subjects, Beirut, pp.115,116.

-Ward, W.(1982). Index of Egyptian Administrtive and Religious Titles of the Middle Kingdom, Beirut, pp.7 n 13, 84 n 695, n 696, n. 697, n, 700, n. 701.

-Weill, R. (1905). <<Sur At.w-n-T.t-HqA et quelques titres analogues du Moyen Empire>>, Recueil des Travaux relatifs à la philologie et à $l$ 'archeologie égyptiennes et assyriennes 27, pp.41-43.

- Wolterman, C.( 1996). <<A vizir of Ramses III visits an oracle >>, Revue d'Egyptologie 47, Paris p.161. 